\title{
The analysis and design of supply chain system based on CAF technology
}

\author{
LIU Re , ZHANG Chunyan \\ Wuxi Professional College of Science and Technology, Wuxi, Jiangsu, China \\ liurewx@163.com
}

\begin{abstract}
Keywords: CAF technology Supply Chain System System Analysis and Design.
\end{abstract}
\begin{abstract}
This paper introduces the basic concepts of CAF technology and takes its system analysis, system design practice to participate in the development of Image Capture system, as example, demonstrated the CAF technical framework for the application of supply chain systems in a distributed system field of practical and good practical value.
\end{abstract}

\section{Introduction}

The Supply chain logistics is capital flow and information flow between production and interrelated departments or business partners what happened, can also be understood as material acquisition, processing material or an intermediate semi-finished products, finished products and then sent to a network user in the hands of a series of business or corporate sector constituted.

CAF (Collaborative Application Framework) is a collection of software components, This set can make use of the network of business partners to collaborate versatility transactions; CAF support collaboration between companies, B2B support collaboration between trading partners. By CAF after platform integration supply chain system not only better fulfill the function of the original system, and other systems at the same time open up channels of cooperation, can provide a more rich, real-time, accurate business functions.

CEF-based supply chain system by CAF machine technology to manufacturers, suppliers, service providers and other supply chain partners together, for each control end to end logistics functions. System further stressed the work of different information systems, and between the various partners are regarded as a market focused on the point of contact, this structure makes the supply chain execution applications have strong scalability, making it very easy to meet new increasing demand partners. This pape takes the system of Image Capture as example to explain how to use its technology to complete the analysis and design of CAF supply chain system.

\section{The Analysis and Design of CAF system}

The Frames of CAF

Image Capture System is a set up for digital camera supply chain system, the system is based on the entire production process of digital cameras to relate to various business partners connected to this system, allowing them to coordinate work in this system, jointly controlled digital camera production, the supply chain relationship shown in Figure 1. 


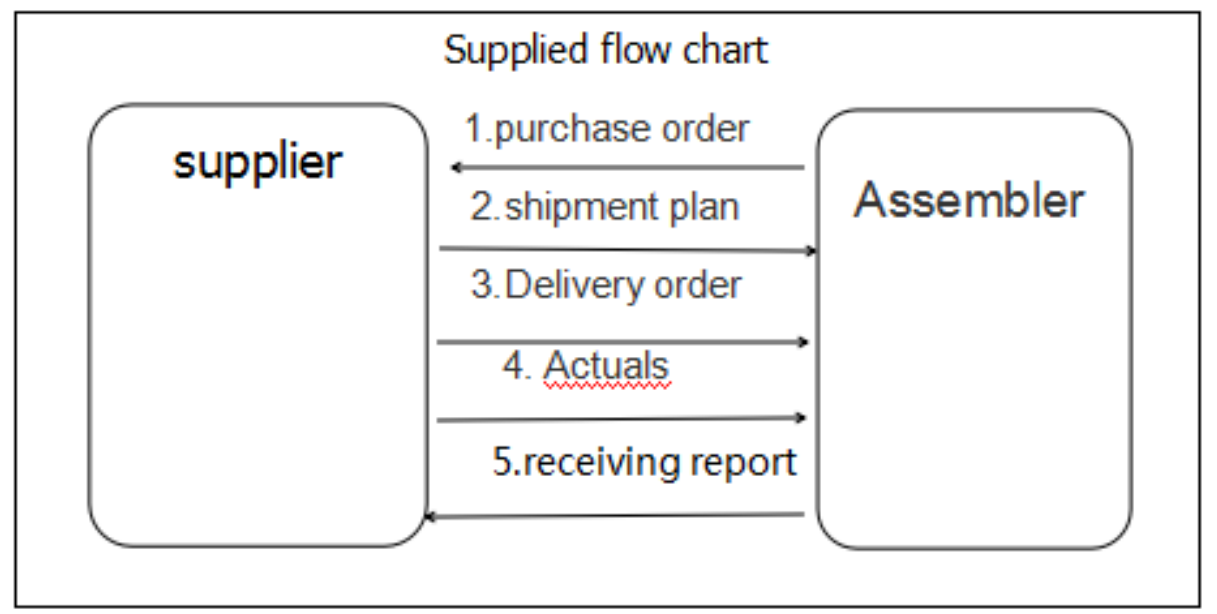

Figure 1. working relationship between supplier and Assembler

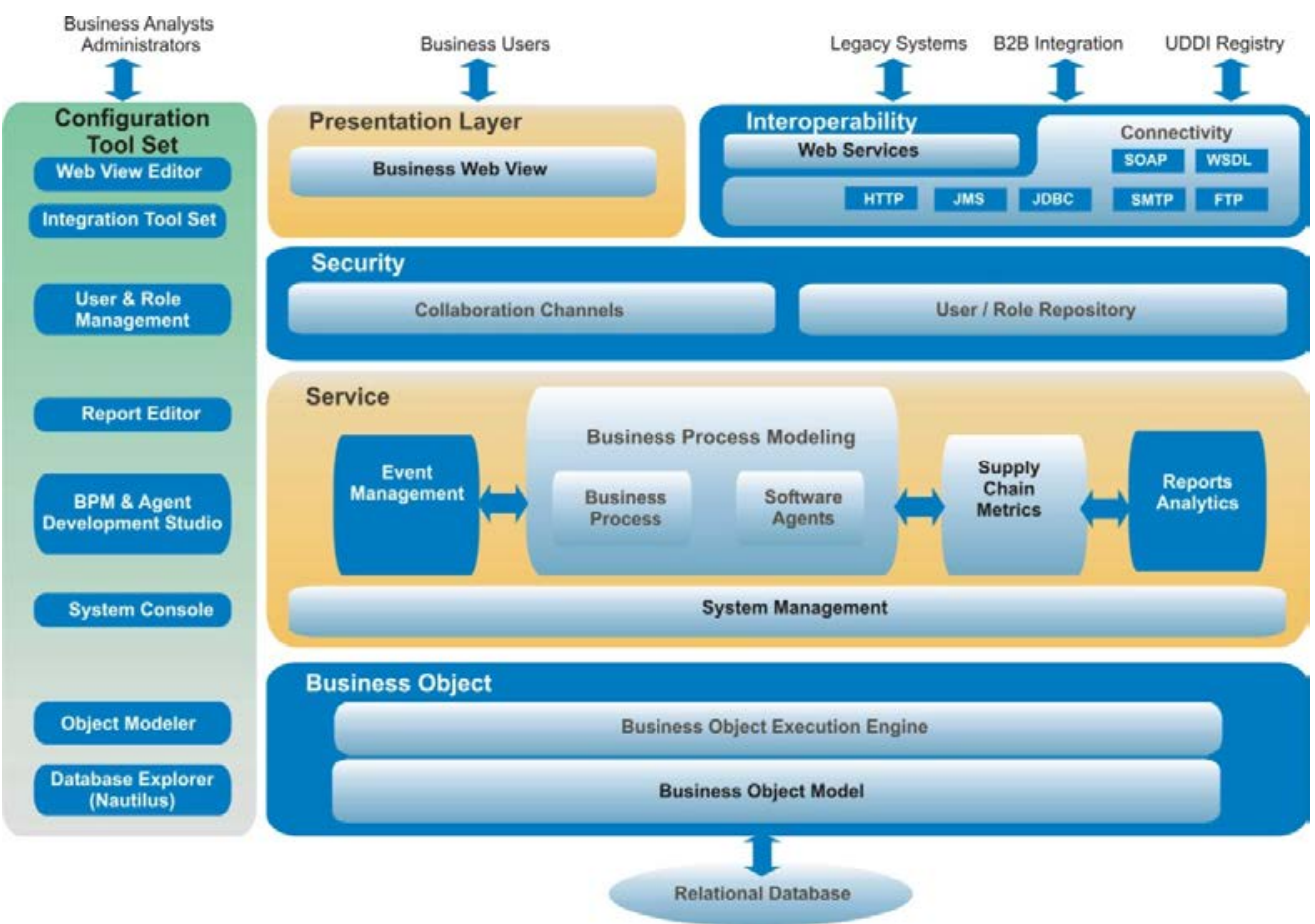

Figure 2.The frame of CAF

Shown above CAF architecture is hierarchical, top to bottom:

Presentation Layer - driven by XSL generator.

Security - there are SSL, enter the security certificate management system, that regardless of what kind of access to the system, the system must be certified by

Service - Events and schedule-driven, primarily for transaction management system, while the supply chain workflow simulation is also done here.

Commercial entities (BO: Business Object) - server components, storage components, views, and so are the BO. Local business entity layer protects the data layer is not affected by the commercial application of the system. No SQL code to handle complex database relationships. XML formatted business entity objects is the only source of data required by the system. This part plays an important feature is a runtime component, which has the capacity to accept forms of business entity object XML query, as well as having received the sentence translated to the relational database operation statement, then begin the actual database operation.

Relational databases - using an Oracle database, the system program is not directly manipulate the database, but the use of the top layer of BO (Business Object) is controlled indirectly. Not need to know the exact location of the relevant data tables. 


\section{The analysis of CAF framework}

1. Based on business entities

- commercial entities and commercial entities engine model allows the graphical definition and configuration of commercial entities.

2. Business process activation

- Business process structure permitted between the actual business processes and systems to achieve a simple executable processes to accelerate the implementation of the mapping.

3. Agent push

- Agent Framework provides the ability to quickly build and configuration of business logic and business integration.

4. Event-driven

- real-time events and alarms generated, and passed out of the system.

5. The system configuration

- promote the concept of "Configuring the system". The system can use the powerful built-in configuration tools to quickly create, does not require any coding.

- The system can continue to modify the minimum cost and re-configured to adapt to the changing business environment.

6. work

- support the integration of the majority of standard communication protocols (Web Services, HTTP, database, COM, sockets, FTP, files, etc).

- make the system and other systems can interact with information, in order to achieve interoperability.

\section{Design of Image Capture System operating environment}

A .CAF framework server

CAF servers are divided into three types: CAF Agent Framework, CAF Web Exchange and CAF System Monitor.

1. CAF Agent Framework

CAF Agent Framework as CAF server 3. The server handles Agents running in the background. These Agents, such as ImportAgent, the main monitor, the background processing system from the information released, so there is no user interface.

2. CAF Web Exchange

CAF Web Exchange server became CAF 1. The server is responsible for user sees, in direct contact with the user interface. In other words, the server is responsible to respond to HTTP posts, and HTTP post here refers to user demand raised by CAF user interface, such as the user clicks a button; the user through the CAF user interface for a particular part to develop a complementary program, etc. .

3. CAF System Monitor

CAF System Monitor also refers to the CAF Server2. This server is used to monitor a variety of components and CAF CAF rely on components.

B.The agents of CAF framework

CAF is based Agents function; CAF in various Agent to perform different tasks, such as email agent, alert agent and so on. All agents are stored in xml configuration file.

One of the major Agent has: UploadAgent, PromoteAgent, AlertAgent ImportAgent and so on.

In Client Agent Case:

How users work through the process Client Agent as shown in Figure 3, from a business partner system to the required data in the form of export XML, converting data into XML format Image Capture system requirements, and then upload the data to Image Capture system temporary data area, the system these data content and format of some aspects of the inspection, the last system in a specific period of time will correct these data into a database system. 


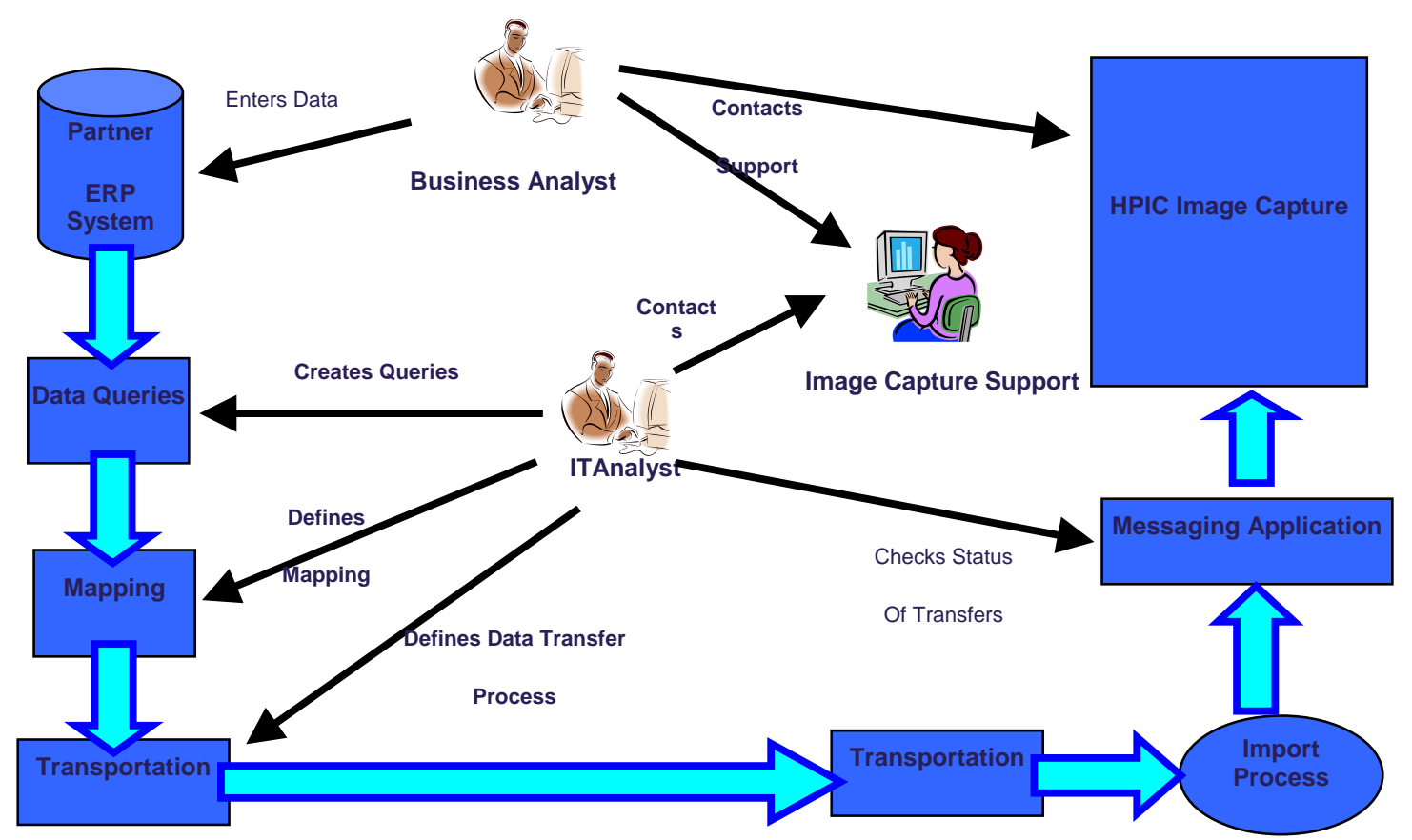

Figure 3. The Process of Client Agent Working

C. CAF framework database

The database system is designed to be based on Oracle. It includes information about the different configuration of CAF Servers, the configuration information can be used to run the system once established. In addition, the database also includes a number of processes within the database, which is responsible for implementing a number of key business processes, such as the system used to calculate the CAF application of different data KPI Engine (Key Performance Indicators Engine, engine key performance indicator) is one such. KPI configured to process data based on different database tables and running, and will reflect the new information by calculating different amounts. But the system is not part of the CAF database directly accessible by the application system function calls, but accessed via Business Object.

\section{Acknowledgements}

Top-notch Academic Programs Project of Jiangsu Higher Education Institutions.

School of science and technology fund projects , <RJ15031>.

\section{References}

[1] Ma Shihua, Lin Yong, Chen Zhixiang supply chain management Beijing: Mechanical Industry Press, 2014: 35-49

[2] Bo Meng J2 EE-based B2B e-commerce platform to build Sichuan: Computer Application Research, 2013: 121 to 124

[3] Benedict instigate agile supply chain and its supporting technologies Nanjing: Southeast University Thesis, 2012: 53 to 67

[4] Wangcheng En supply chain logistics and information flow management. Beijing: China Management Science, 2013: 16 to 23

[5] Chang manufacturing resource planning MRP-II and its application Beijing: Tsinghua University Press, 2015: 13-16 